investigate the use of breath analysis as a non-invasive and simpler means of diagnosing HE, cirrhosis and harmful drinking.

Methods A bespoke breath-sampling device was used to sample one litre of breath through adsorbent tubes from patients with alcoholrelated cirrhosis with $(n=11)$ and without $H E(n=23)$, non-alcoholic cirrhosis without HE $(n=19)$, harmful drinkers without cirrhosis $(n=7)$, inflammatory respiratory disease $(n=18)$, and healthy controls $(n=15)$. Compounds trapped on these tubes were released via thermal desorption and analysed by gas chromatography mass spectrometry for separation and detection. Multivariate discriminant analysis was used to identify volatile organic compounds to differentiate patients according to disease status and build models for disease classification. Results Models based on the presence or absence of volatiles were tested in the patient groups. HE was correctly classified in $91.0 \%$ of patients with alcoholic cirrhosis. Patients with cirrhosis could be discriminated from those without cirrhosis with 100\% accuracy in drinkers. In patients without clinical signs of $\mathrm{HE}$, alcohol was correctly predicted as the underlying cause of cirrhosis in $82.6 \%$ of patients and non-alcoholic causes of cirrhosis were correctly determined in $84.2 \%$ of patients. Non-alcoholic cirrhosis, alcoholic cirrhosis, and harmful drinking could also be discriminated from healthy controls with a sensitivity of $89.5 \%, 97.1 \%$ and $100 \%$, respectively.

Conclusion Breath volatiles can be used to aid the diagnosis of HE, cirrhosis, and harmful levels of drinking, therefore breath testing may offer a means to detect liver conditions non-invasively at earlier and more treatable stages.

Competing interests None declared.

\section{PWE-288 SPONTANEOUS BACTERIAL PERITONITIS: PREVALENCE ON ADMISSION TO A TERTIARY CENTRE AND SUBSEQUENT OUTCOME}

doi:10.1136/gutjnl-2012-302514d.288

T Bugeja, * W T Gelson, W J H Griffiths. Department of Hepatology, Addenbrooke's Hospital, Cambridge, UK

Introduction Spontaneous bacterial peritonitis (SBP) is a sinister complication of cirrhosis associated with poor survival (approximately $38 \%$ at 1 year $^{1}$ ). However, a diagnosis of SBP does not represent, in its own right, an indication for liver transplantation in the UK under current listing criteria.

Methods We sought to investigate the prevalence and subsequent mortality in patients with an admission diagnosis of SBP. We retrospectively identified 366 consecutive cases admitted with ascites to our liver unit during the calendar year 2009. Of these 271 $(74 \%)$ patients underwent diagnostic paracentesis at time of admission and were included for further analysis.

Results Of the 271 cases, 26 (9.6\%) were diagnosed with SBP at admission on the basis of an ascitic fluid white cell count above 250 cells $/ \mathrm{mm}^{3}$ (25 cases). Patients with positive cultures but no white cell response were only included if symptomatic (one case). Alcohol was the underlying aetiology in 17 cases (65.4\%) and clinical presentations were as follows: routine paracentesis (14 cases), associated pleural effusion (four cases), variceal haemorrhage (three cases), abdominal pain (two cases), jaundice (two cases), encephalopathy (one case). Ascitic culture on two patients grew gramnegative bacilli, both resistant to quinolones being used for prophylaxis. One ascitic culture grew Lactobacillus spp. and one a mixed growth of Enterococcus faecalis and gram-positive cocci. Three patients $(11.5 \%)$ died during the index admission. Three patients $(11.5 \%)$ had successfully undergone liver transplantation and were alive 6 months after admission. Overall mortality at 6 months from an admission diagnosis of SBP was $50 \%$.
Conclusion SBP is not uncommon in cirrhotic patients with ascites, can often present silently and is associated with high mortality. Resistance to standard quinolone prophylaxis and isolation of grampositive bacteria are more recent phenomena in this group of patients. All cirrhotics admitted with significant ascites should undergo diagnostic paracentesis to exclude SBP and assessment for liver transplantation must be an urgent consideration in appropriate candidates. Listing criteria may need to be revised to include SBP as a standard indication.

Competing interests None declared.

\section{REFERENCE}

1. Tito $\mathbf{L}$, et al. Recurrence of spontaneous bacterial peritonitis in cirrhosis: frequency and predictive factors. Hepatology 1988;8:27-31.

\section{PWE-289 CRITICAL ILLNESS EARLY WARNING SCORES RETAIN ACCURACY IN PATIENTS WITH LIVER DISEASE-AN ANALYSIS OF 182000 INPATIENT OBSERVATION SETS}

doi:10.1136/gutjnl-2012-302514d.289

${ }^{1} \mathrm{~T}$ Hydes, ${ }^{2} \mathrm{P}$ Schmidt, ${ }^{3} \mathrm{G}$ B Smith, ${ }^{4} \mathrm{D}$ Prytherch, ${ }^{1} \mathrm{R} \mathrm{J}$ Aspinall. ${ }^{1}$ Department of Gastroenterology and Hepatology, Queen Alexandra Hospital, Portsmouth, UK; ${ }^{2}$ Acute Medicine, Queen Alexandra Hospital, Portsmouth, UK; ${ }^{3}$ School of Health \& Social Care, Bournemouth University, Bournemouth, UK; ${ }^{4}$ University of Portsmouth Centre for Healthcare Modelling and Informatics, Queen Alexandra Hospital, Portsmouth, UK

Introduction Aggregate weighted track and trigger systems (AWTTS) can identify critically ill patients at high risk of mortality. However, these scores use features of the Systemic Inflammatory Response Syndrome (SIRS) that may be altered in liver disease such as resting tachycardia and hypotension secondary to hyperdynamic circulation, blunted pyrexial response or hyperventilation due to encephalopathy. These scores have not been evaluated in patients with liver disorders. We therefore examined whether a range of AWTTS, including the VitalPac Early Warning Score (ViEWS), retain accuracy in liver disease.

Methods Clinical observations were recorded on a computerised database (VitalPac) for all admissions between July 2006 and April 2011 in a large hospital serving a population of 650000 . Adults assigned International Classification of Diseases (ICD-10) codes for liver disease, either as primary or comorbid diagnosis, were identified. ViEWS scores of $0-19^{1}$ were allocated to all vital sign sets. Each set contained: date/time, pulse, blood pressure, respiration, temperature, neurological status using either the Alert-Verbal-Painful-Unresponsive scale or Glasgow Coma Score, pulse oximetry and use of supplemental oxygen. Datasets were analysed with respect to number of patients needed to be seen by a doctor if escalation occurred at that score and inpatient mortality within $24 \mathrm{~h}$.

Results We identified 44328 observation sets for patients with a primary liver diagnosis (PLD) code of which 519 (1.17\%) were followed by death within $24 \mathrm{~h}$. For those with a non-primary liver diagnosis (NPLD) 138217 observations were made and 1157 (0.84\%) resulted in death. There were no differences at any ViEWS score in the prediction of 24-h mortality and number needed to be seen for patients assigned a PLD or NPLD, compared to all adult hospital admissions (Abstract PWE-289 figure 1). The area under the receiveroperating characteristics curve (95\% CI) was 0.886 for all patients and 0.888 and 0.883 for those with PLD and NPLD respectively.

Conclusion Using an electronic database of all clinical observations and diagnostic codes, we found the accuracy of predicting death within $24 \mathrm{~h}$ was retained in the presence of liver disease regardless of primary or secondary diagnosis. We have now expanded this work to include analysis of 34 additional AWTTS scores and distinct clinical presentations of hepatic disorders.

Competing interests T Hydes: None declared, P Schmidt Shareholder with: Director of a company with a minority shareholding in the development of Learning Clinic 